\title{
On Conjugacy of $p$-gonal Automorphisms of Riemann Surfaces
}

\author{
Grzegorz GROMADZKI \\ Institute of Mathematics \\ University of Gdańsk \\ Wita Stwosza 57 \\ 80-952 Gdańsk — Poland \\ greggrom@math.univ.gda.pl
}

Received: January 8, 2007

Accepted: August 30, 2007

\begin{abstract}
The classical Castelnuovo-Severi theorem implies that for $g>(p-1)^{2}$, a $p$-gonal automorphism group of a cyclic $p$-gonal Riemann surface $X$ of genus $g$ is unique. Here we deal with the case $g \leq(p-1)^{2}$; we give a new and short proof of a result of González-Diez that a cyclic p-gonal Riemann surface of such genus has one conjugacy class of $p$-gonal automorphism groups in the group of automorphisms of $X$.

Key words: automorphisms of Riemann surfaces, fixed points, ramified coverings of Riemann surfaces, hyperellipticity.

2000 Mathematics Subject Classification: Primary 30F10; Secondary 14H37, 14 H55.
\end{abstract}

\section{Introduction}

A compact Riemann surface $X$ of genus $g \geq 2$ is said to be cyclic $p$-gonal if there is an automorphism $\varphi$ of $X$ of order $p$ such that the orbit space $X / \varphi$ is the Riemann sphere. Such automorphism is called $p$-gonal automorphism and it gives rise to a ramified covering of the Riemann sphere by $X$ with $p$ sheets. So Castelnuovo-Severi theorem [4] asserts that for $g>(p-1)^{2}$, the group generated by a $p$-gonal automorphism of a Riemann surface of genus $g$ is unique as was mentioned by Accola in [1]. Here we

Supported by the grant SAB2005-0049 of the Spanish Ministry of Education and Sciences and by MTM2005-01637. 
prove that for $g \leq(p-1)^{2}$, a cyclic $p$-gonal Riemann surface has one conjugacy class of $p$-gonal automorphism groups in the group $\operatorname{Aut}(X)$ of automorphisms of $X$. This result has been proved using different techniques by González-Diez in [5].

We shall use combinatorial methods based on the Riemann uniformization theorem and combinatorial theory of Fuchsian groups as in [6] (see also [3]), where the reader can find necessary notions and facts.

\section{On fixed points of automorphisms of Riemann surfaces}

By the Riemann uniformization theorem an arbitrary compact Riemann surface of genus $g$ can be represented as the orbit space $\mathcal{H} / \Gamma$, where $\mathcal{H}$ is the upper half plane and $\Gamma$ is a Fuchsian surface group with signature $(g ;-)$. A group of automorphisms of a surface so given can be presented as $\Lambda / \Gamma$ for some Fuchsian group $\Lambda$. So the Riemann Hurwitz formula gives at once the following easy but useful result.

Lemma 1.1. A Riemann surface $X=\mathcal{H} / \Gamma$ is cyclic $p$-gonal for a prime $p$ if and only if there exists a Fuchsian group with signature $(0 ; p, . . s ., p)$, where $s=2(g+p-1)$ / $(p-1)$, containing $\Gamma$ as a normal subgroup of index $p$.

Observe that a $p$-gonal automorphism of a Riemann surface of genus $g$ has $2(g+p-1) /(p-1)$ fixed points. We shall use the following theorem of Macbeath [7] concerning fixed points of automorphisms of Riemann surfaces.

Theorem 1.2. Let $X=\mathcal{H} / \Gamma$ be a Riemann surface with the automorphism group $G=\Lambda / \Gamma$ and let $x_{1}, \ldots, x_{r}$ be a set of elliptic canonical generators of $\Lambda$ whose periods are $m_{1}, \ldots, m_{r}$ respectively. Let $\theta: \Lambda \rightarrow G$ be the canonical epimorphism. Then the number $F(\varphi)$ of points of $X$ fixed by a nontrivial element $\varphi$ of $G$ is given by the formula

$$
F(\varphi)=\left|N_{G}(\langle\varphi\rangle)\right| \sum 1 / m_{i},
$$

where $N$ stands for the normalizer and the sum is taken over those $i$ for which $\varphi$ is conjugate to a power of $\theta\left(x_{i}\right)$.

Finally we shall use the following easy

Lemma 1.3. Let $G$ be a finite group of order bigger than $p^{2}$ generated by two elements $a, b$ of prime order $p$. Then for the normalizer $N$ of the group generated by $a$, $|N| \leq|G| / p$.

Proof. Clearly no nontrivial power of $b$ belongs to $N$ since otherwise $|G| \leq p^{2}$. So $[G: N] \geq p$. 


\section{On $p$-gonal automorphisms of Riemann surfaces}

As we mentioned before for $g>(p-1)^{2}$, a $p$-gonal automorphism group of a cyclic $p$-gonal Riemann surface of genus $g$ is unique. Here we deal with $g \leq(p-1)^{2}$.

Theorem 2.1. A cyclic p-gonal Riemann surface of genus $g \leq(p-1)^{2}$, has one conjugacy class of p-gonal automorphism groups in the group $\operatorname{Aut}(X)$ of automorphisms of $X$.

Proof. Let $X$ be a cyclic $p$-gonal Riemann surface of genus $g \leq(p-1)^{2}$ and let $\left\langle a_{1}\right\rangle, \ldots,\left\langle a_{m}\right\rangle$ be representatives of all conjugacy classes of $p$-gonal automorphism groups. By the Riemann uniformization theorem $X=\mathcal{H} / \Gamma$ and by a Sylow theorem $a_{1}, \ldots, a_{m}$ can be assumed to belong to a $p$-subgroup of $\operatorname{Aut}(X)$. Assume, to get a contradiction, that $m \geq 2$, denote $a_{1}=a, a_{2}=b$, and let $G=\langle a, b\rangle$. Then $G=\Lambda / \Gamma$, where $\Lambda$ is a Fuchsian group with signature $\left(h ; m_{1}, \ldots, m_{r}\right)$. Let $\theta$ be the canonical projection of $\Lambda \rightarrow G$.

We shall show first that $G$ has order $p^{2}$. In contrary assume that $|G|=n>p^{2}$. Then, by Lemma 1.3 and Theorem 1.2, every period of $\Lambda$ produces at most $n / p^{2}$ fixed points of $a$ or $b$ and therefore in particular

$$
4(g+p-1) /(p-1) \leq r n / p^{2} .
$$

Now for $h \neq 0$, the area $\mu(\Lambda)$ of $\Lambda$ satisfies $\mu(\Lambda) \geq 2 \pi r(p-1) / p$ and so, by (1) and the Hurwitz-Riemann formula, $2 g-2 \geq 4 p(g+p-1) \geq 12(g+2)$. Thus $h=0$. But then $r \geq 3$.

First, let $r \geq 4$. Then $\mu(\Lambda) \geq 2 \pi(-2+r(p-1) / p)$ and so, by the Hurwitz-Riemann formula, $g \geq n r(p-1) / 2 p-n+1 \geq n(p-2) / p+1 \geq n / 3+1$. On the other hand the Hurwitz-Riemann formula and (1) gives also $2 g-2 \geq-2 n+4 p(g+p-1) \geq-2 n+12 g$ and so $g<n / 5$, a contradiction.

Now let $r=3$. Since $a$ and $b$ can not be simultaneously conjugate to a power of some $\theta\left(x_{i}\right)$, only one proper period produces fixed points in $a$ or in $b$ by Theorem 1.2; assume that this is the case for $a$. Then since $a$ and $b$ have the same number of fixed points, the remaining two proper periods may produce at most $n / p^{2}$ fixed points in $b$. So (1) actually becomes

$$
4(g+p-1) /(p-1) \leq 2 n / p^{2} .
$$

But for $p \geq 5, \mu(\Lambda) \geq 2 \pi(-2+3(p-1) / p)$. Thus

$$
\begin{aligned}
4 \pi(g-1) & =n \mu(\Lambda) \\
& \geq 2 \pi(-2 n+3 n(p-1) / p) \\
& \geq 2 \pi(-2 n+6 p(g+p-1))
\end{aligned}
$$

and so $g \leq n / 14$. On the other hand, by the Hurwitz-Riemann formula $n=4 \pi(g-1) / \mu(\Lambda) \leq 2 p(g-1) /(p-3)$ which gives $g \geq n / 5$, a contradiction. 
For $p=3$, a period of $\Lambda$ is at least 9 since $(0 ; 3,3,3)$ is not a signature of a Fuchsian group. But then $\mu(\Lambda) \geq 4 \pi / 9$ and therefore by the Hurwitz-Riemann formula $g \geq n / 9$, while by $(2) g \leq n / 9-2$, a contradiction.

So we can assume that $G$ has order $p^{2}$ and therefore $G=\mathrm{Z}_{p} \oplus \mathrm{Z}_{p}$. Here $\Lambda$ has signature $(h ; p, . \stackrel{r}{.}, p)$, with $h=0$ by the Hurwitz-Riemann formula. But then for $r \geq 5, \mu(\Lambda) \geq 2 \pi(3 p-5) / p$ and so the Hurwitz-Riemann formula gives $g \geq p(3 p-5) / 2+1$ which is bigger than $(p-1)^{2}$. So $r \leq 4$.

However, for $r=3$, there is a Fuchsian group $\Lambda^{\prime}$ with signature $(0 ; 3,3, p)$ containing $\Lambda$ as a subgroup and $\Gamma$ as a normal subgroup by [8] and by Theorem 5.2 (i) of [2] respectively. Furthermore by N6 of [2], all canonical generators of $\Lambda$ are conjugate in $\Lambda^{\prime}$ and so all $p$-gonal automorphism groups of our surface are conjugate in $\Lambda^{\prime} / \Gamma \subseteq \operatorname{Aut}(X)$, a contradiction.

The case $r=4$ is similar. Here each $\theta\left(x_{i}\right)$ is conjugate to a nontrivial power of $a$ or $b$ since otherwise $a$ and $b$ would have at most $3 p$ fixed points in total, by Theorem 1.2, while on the other hand they should have $4 p$ such points by Lemma 1.1, since by the Hurwitz-Riemann formula the genus of the corresponding surface equals $(p-1)^{2}$. So, for some permutation $\sigma$,

$$
\theta\left(x_{\sigma(1)}\right)=a^{\alpha}, \quad \theta\left(x_{\sigma(2)}\right)=a^{-\alpha}, \quad \theta\left(x_{\sigma(3)}\right)=b^{\beta}, \quad \theta\left(x_{\sigma(4)}\right)=b^{-\beta} .
$$

But then the mappings

and

$$
\theta\left(x_{1}\right) \longmapsto \theta\left(x_{2}\right), \quad \theta\left(x_{2}\right) \longmapsto \theta\left(x_{1}\right), \quad \theta\left(x_{3}\right) \longmapsto \theta\left(x_{4}\right), \quad \theta\left(x_{4}\right) \longmapsto \theta\left(x_{3}\right)
$$

$$
\theta\left(x_{1}\right) \longmapsto \theta\left(x_{4}\right), \quad \theta\left(x_{2}\right) \longmapsto \theta\left(x_{3}\right), \quad \theta\left(x_{3}\right) \longmapsto \theta\left(x_{2}\right), \quad \theta\left(x_{4}\right) \longmapsto \theta\left(x_{1}\right)
$$

induce automorphisms of $G$. So by N4 of [2], we obtain that a nontrivial power of $a$ is conjugated to a nontrivial power of $b$ in $\operatorname{Aut}(X)$. This is a contradiction which completes the proof.

Acknowledgement. The author is grateful to Professor Antonio F. Costa for his helpful comment and to the anonymous referee for his/her useful suggestions and for pointing out some minor gaps in the proof of the main theorem.

\section{References}

[1] R. D. M. Accola, On cyclic trigonal Riemann surfaces, I, Trans. Amer. Math. Soc. 283 (1984), no. $2,423-449$.

[2] E. Bujalance, F. J. Cirre, and M. Conder, On extendability of group actions on compact Riemann surfaces, Trans. Amer. Math. Soc. 355 (2003), no. 4, 1537-1557.

[3] E. Bujalance, J. J. Etayo, J. M. Gamboa, and G. Gromadzki, Automorphism groups of compact bordered Klein surfaces: A combinatorial approach, Lecture Notes in Mathematics, vol. 1439, Springer-Verlag, Berlin, 1990. 
[4] G. Castelnuovo, Sulle serie algebriche di gruppi di punti appartenenti ad una curve algebraica, Rend. Real Accad. Lincei (5) 15. Memorie scelte, p. 509.

[5] G. González-Diez, On prime Galois coverings of the Riemann sphere, Ann. Mat. Pura Appl. (4) 168 (1995), 1-15.

[6] A. M. Macbeath, Discontinuous groups and birational transformations, Dundee Summer School (Univ. of St. Andrews, 1961).

[7] _ Action of automorphisms of a compact Riemann surface on the first homology group, Bull. London Math. Soc. 5 (1973), 103-108.

[8] D. Singerman, Finitely generated maximal Fuchsian groups, J. London Math. Soc. (2) 6 (1972), no. $1,29-38$. 